\title{
Fast Registration Algorithm for 3D Medical Images using Double Density Dual Tree Complex Wavelet Transform and Bi-directional Prediction
}

\author{
Girija J. ${ }^{1}$, Dr. Krishna Murthy G.N. ${ }^{2}$ and Dr. P. Chenna Reddy ${ }^{3}$ \\ ${ }^{1}$ Research Scholar, Department of CSE, JNTU \\ Ananthapuram, AP, India \\ 2 Principal, BNMIT, VTU \\ Bangalore Karnataka, India \\ ${ }^{3}$ Professor, JNTU, Department of CSE, \\ Ananthapurm, Andra Pradesh, India
}

\begin{abstract}
This paper discuss about 3D-3D CT-MRI image registration carried out using Double Density Dual Tree Complex Wavelet Transform (DD-DTCWT) with bi-directional prediction algorithm considering $2 \mathrm{D}$ frames. The $3 \mathrm{D}$ images of CT and MRI are sub sampled by considering odd frames. The selected odd frames are registered by computing the DD DTCWT features and mutual information metric. The registered 2D frames for which transformation parameters that are estimated are used in registering the even frames with bidirectional prediction algorithm. The algorithm is designed to be reconfigurable to select transformation type such as DWT, DTCWT, DD-DTCWT. The wavelet sub bands selection logic is set as control parameters between speed and registration accuracy. The registration algorithm performance is estimated considering mutual information and joint entropy with improvement of $10 \%$ and $6 \%$ respectively.
\end{abstract}

Keywords: $3 D$ Image Registration, CDDWT, Mutual Information, Optimum Features, Multimodal images.

\section{Introduction}

With advances in medical imaging technologies in 3D visualization of anatomy, detection and monitoring of tumors has given edge over existing technologies for doctors in treating patients. More than forty 3D images and 20 different structures are analyzed in patient treatment. Determining the tumors avoiding critical structures, knowing the patterns of tumors, motions and changes in motions during course of treatment is time consuming and demands highly accurate registration techniques of multimodal images. The medical equipment used in clinical workflow need to be integrated with 3D/4D registration techniques considering all uncertainties, breathing motion, respiration sorting and in-room imaging. The objects in medical images change with time due to elasticity and abnormalities leading to motion of objects in all three directions that are captured in 3D images. In 3D registration, registration should be carried on every slice of 3D data set as there could be non-uniform movement in the structures due to non-rigid objects and removal or changes in objects due to clinical intervention[1][2]. In feature based image registration, features such as regions, edges and points are sensed from the input image and compared with the features in reference image [3]. A transformation is carried out so as to minimize the distance between these features. In [4] new unique approach to compute a stable homography between a pair of images using iterative refinement of transformation parameters, an iterative image registration approach to compute accurate and stable transformation parameters. In [5] to reduce the time for feature detecting, SURF technique is mostly used algorithm as it is the fastest descriptor. RANSAC is applied for outlier elimination and to robustly estimate the best fitting homography, and at end homography transformation is used as transformation model. In [6] Registration of MRI and CT images by selection of tibia and femur features from the binary images and affine transformation, validation of the algorithm is carried out by considering correlation coefficient. In [7], preprocessing techniques such as geometric feature based segmentation dynamic threshold method, feature extraction techniques such as automated trunk slices extraction and transformation technique like multithread iterative closest point are used for registration. Validation metrics such as negative normalization correlation and Euclidean distance error are computed for registering PET and CT images. Huizhong Chen and Nick Kingsbury [7] have used Dual Tree Complex Wavelet Transform (DTCWT) coefficients to align images by considering phase information of coefficients. The DTCWT front-end filter is shift invariance and directional selective and hence the registration algorithm is robust to local mean and contrast changes in 
images to be registered. In [8], two step registration processes is carried out by considering the sub band information computed using DTCWT. Coarse level registration is carried out by considering edges and cross correlation function from low frequency bands and the registration is improved by considering mutual information from six orientation bands. In [9], registration of medical images is carried out by computing DTCWT sub bands for key point's selection, Hausdorff distance for similarity measurement between key points and Niche particle swarm optimization for affine transformation. Evaluation metrics such as correlation coefficient, normal mutual information, mean square error and time are used. Most of the methods discussed use affine transformation as deformation model, feature selection and similarity checking based on mutual information. Accuracy in registration algorithm depends on selection of object function and transformation model. In [our paper], an improved method of 3D image registration is presented by computing the mutual information from low pass and high pass DTCWT sub bands computed independently and registering the MRI and CT images. One of the limitations of DTCWT is the redundancy in the sub bands, for level-1 3D DTCWT computation there arises 64 sub bands of which 8 of them are low pass bands and 56 of them are high pass bands. Processing all these sub bands for computation of mutual information increases computation complexity as well as computation time. In order to reduce the complexity Complex Double Density Wavelet Transform (CDDWT) is presented in this work to perform $3 \mathrm{D}$ image registration.

Section 2 discusses 3D CDDWT algorithm, Section 3 discusses proposed image registration algorithm, Section 4 discusses the results and Section 5 is conclusion.

\section{Complex Double Density Wavelet Transform}

Discrete Wavelet Transform (DWT) for signal and image processing has found wider adoptability in the last two decades since the time Continuous Wavelet Transform (CWT) was first introduced by Grossman and Morlet in 1982 [10]. Mallat and Meyer in 1988 [11] presented multiresolution analysis for real world signal processing and analysis and devised DWT as fast processing algorithm. With DWT being a critical sampled version of CWT, analyzing real world signal was much easier using DWT. Daubechies in 1988 [12] presented new family of wavelet for multiresolution analysis of real world data using DWT that is faster and orthogonal. DWT although being a power tool for real work signal analysis has been observed to have limitations such as shift sensitive [13], very poor directional selective [14] and does not contain phase information[15]. In order to overcome shift sensitive limitations undecimated DWT was proposed in [16] which is similar to DWT without decimation. Kingsbury and Magarey [17] have improvised Dual Tree Wavelet Transform to capture directionality in six sub bands by using analytic filters. In [18] Complex Wavelet Transform (CWT) was proposed to overcome all the three limitations and termed it as nonredundant, directional CWT. The CWT by Felix C. A. Fernandes [18] is flexible and can be generated by using real value input signal projected on complex sub space and computing DWT based on the projection using any generic DWT. Further in [17] a new transform termed as Complex Double Density Wavelet Transform (CDDWT) is proposed based on Selesnick Double Density DWT, this new transform is shift sensitive and has low redundancy. The double density DWT defined by Selesnick consists of three filters $h^{0}, h^{1}$ and $h^{2}$ that generates nine sub bands for a input image and these nine sub bands are denoted by $h_{y}{ }^{i} h_{x}{ }_{x}, i, j$ $\in\{0,1,2\}$. The subscript $x$ and $y$ indicate processing of data along row and column respectively. Felix introduced an interleaving process as shown in Figure 1 that is operated on nine sub bands to generate four sub bands denoted as $\mathrm{HH}_{\mathrm{DD}}, \mathrm{HL}_{\mathrm{DD}}, \mathrm{LH}_{\mathrm{DD}}$ and $\mathrm{LL}_{\mathrm{DD}}$.

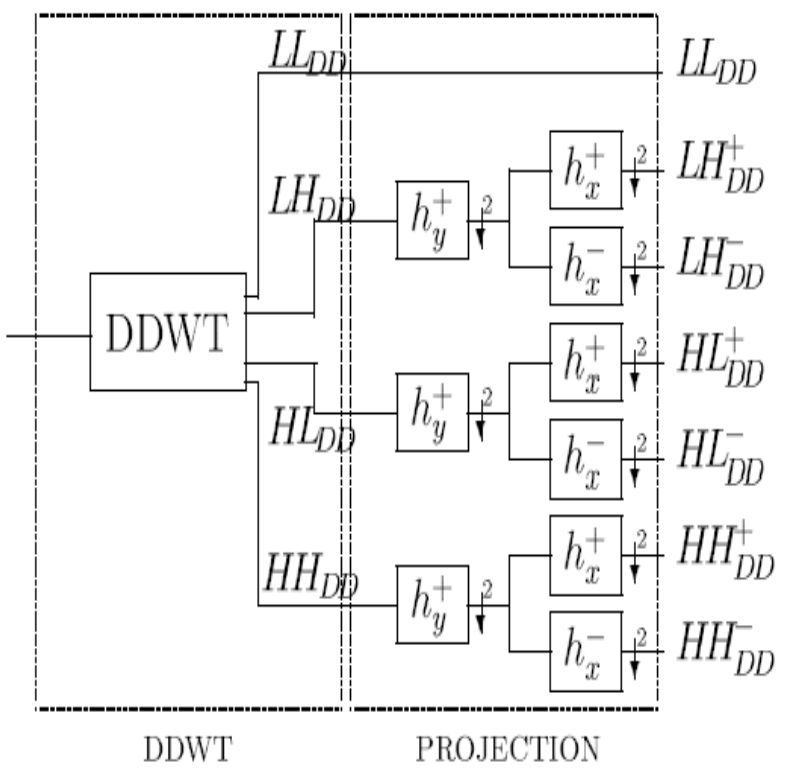

Fig. 1 CDDWT analysis filter bank

These four sub bands are mathematically represented as in Eq. (1), (2) and (3),

$$
\begin{aligned}
& H H_{D D}(m, n)= \begin{cases}h_{y}^{2} h_{x}^{2}\left(\frac{m}{2}, \frac{n}{2}\right) & \text { m even } n \text { even } \\
h_{y}^{2} h_{x}^{1}\left(\frac{m}{2}, \frac{n-1}{2}\right) & \text { m even, } n \text { odd } \\
h_{y}^{1} h_{x}^{2}\left(\frac{m-1}{2}, \frac{n}{2}\right) & \text { modd, } n \text { even } \\
h_{y}^{1} h_{x}^{1}\left(\frac{m-1}{2}, \frac{n-1}{2}\right) & \text { modd }, n \text { odd }\end{cases} \\
& H L_{D D}(m, n)=\left\{\begin{array}{cl}
h_{y}^{2} h_{x}^{0}\left(\frac{m}{2}, n\right) & \text { meven } \\
h_{x}^{0}\left(\frac{m-1}{2}, n\right) & \text { modd }
\end{array}\right.
\end{aligned}
$$




$$
L H_{D D}(m, n)= \begin{cases}h_{y}^{0} h_{x}^{2}\left(m, \frac{n}{2}\right) & n \text { even } \\ h_{y}^{0} h_{x}^{1}\left(m, \frac{n-1}{2}, n\right) & n \text { odd }\end{cases}
$$

The LLDD sub band is made up of only one sub band represented by $\mathrm{h}_{\mathrm{y}}^{0} \mathrm{~h}_{\mathrm{x}}^{0}$. The $\mathrm{HH}_{\mathrm{DD}}$ sub band has four times more coefficients than that of HH sub band of DWT and captures $+45^{0}$ and $-45^{\circ}$ orientations. The $\mathrm{HL}_{\mathrm{DD}}$ and $\mathrm{LH}_{\mathrm{DD}}$ sub band comprises of twice more coefficients than the HL and LH sub band of DWT and captures $\pm 15^{\circ}$ and $\pm 75^{\circ}$ directional feature. Comparing the redundancy factor between DDDWT and CDDWT, the transform domain redundancy is 4 and 2.67 respectively. The CDDWT which is shift insensitive, directional and complex transforms has the redundancy factor of $\left(3^{\mathrm{m}}-1\right) /\left(2^{\mathrm{m}}-1\right)$ where $\mathrm{m}$ represents dimensions. The non-redundant complex wavelet transform is very much suitable for image processing applications as it is non-redundant, directional and has explicit phase information in the sub bands.

\section{$2.1 \quad$ Double Density DTCWT based Registration}

The advantages of DD DTCWT are that in addition to six orientations, the redundancy factor in number of wavelet domain coefficients are limited to 2.67 and hence the number of pixels for processing are limited as compared with DTCWT wavelet domain coefficients. Fig. 2 presents the complex double density dual tree wavelet sub bands compared with dual tree DWT sub bands. As there are localization of orientations, feature extraction from both MRI and CT data will be improved and results in accurate matching process leading to efficient registration algorithm.

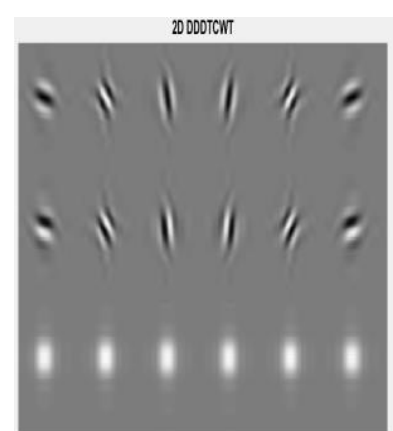

(a)

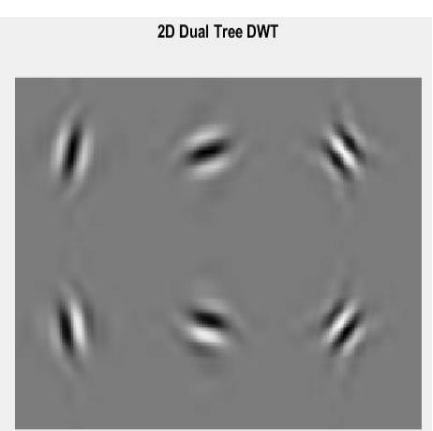

(b)
Fig.2 Comparison of orientations in wavelet sub bands (a) DD DTCWT (b) DT DWT

For evaluation of Image registration algorithm standard data sets from Vanderbilt University with prior permission from Creative Commons, 171 Second Street, Suite 300, San Francisco, California, 94105, USA that comprises of 20 sets of MRI and CT data with 52 frames per 3D data is considered for analysis. The MRI (fixed data) and CT (moving data) is transformed into DD DTCWT sub bands considering 10-tap symmetric filter coefficient and the low pass sub bands of fixed image and moving image. DD DTCWT is computed on every frame of 3D input data which generates 4 low pass sub bands (of which 2 are real and 2 are imaginary) and 32 high pass sub bands (of which 16 of them are real and 16 of them are imaginary). For image registration a reconfigurable logic is designed that allows the user to select either the real sub bands or imaginary sub bands for registration. In addition the reconfigurable logic allows selection of sub bands based on required orientations. The reconfigurable logic functionality is presented in Table 1 .

Table 1. Reconfigurable logic for wavelet mode selection

\begin{tabular}{|l|l|l|}
\hline $\begin{array}{l}\text { Select } \\
\text { Input }\end{array}$ & Selected Bands & $\begin{array}{l}\text { Wavelet } \\
\text { Mode }\end{array}$ \\
\hline 000 & $\begin{array}{l}\text { 1 LL sub band, 3 HH sub } \\
\text { bands }\end{array}$ & DWT \\
\hline 001 & $\begin{array}{l}\text { 2 LL sub band, all HH } \\
\text { sub bands }\end{array}$ & DT CWT \\
\hline 010 & All real sub bands & DD DTWT \\
\hline 011 & All sub bands & DD DTCWT \\
\hline
\end{tabular}

The reconfigurable logic is also designed to select the frames for which registration is to be carried out. In this work, every alternate frame is selected for image registration and the intermediate frames are obtained by bidirectional prediction.

The positions of each sub band in terms of row, column and frame of the 3D images are computed first in terms of global coordinate system. The global reference positions of both MRI and CT images are identified. The transformation parameters are computed by matching the intensity levels of MRI and CT image. This step is iterative and is repeated until best transformation matrix is obtained by setting the optimization and matching metric. Matching metric is either mutual information (Butz and Thiran, 2001, Phim et al 2003, Plumin et al, 204, Maes et al, 2003) or mean square error algorithm. Woods in 1992 introduced the Mates mutual registration measure for multimodality images based on Shannon entropy and probability of cooccurrence measure. Mattes algorithm works better if the matching is carried out on images with features instead of intensities. The Mattes Mutual Information (MMI) metric for DTCWT sub bands are computed as in Eq. (4a),

$I(A, B)=H(A)-H(B \mid A)=H(B)-H(A \mid B)$

Where

$H(B \mid A)=-\sum_{a, b} p(a, b) \log p(b \mid a)=H(A, B)-H(A)$

represents the conditional entropy which is computed by considering the average of the entropy of image sub band $\mathrm{B}$ for each of the intensity in sub band $\mathrm{A}$. H(A) and H(B) 
(shown in Eq. (4b)) represents the individual image entropies of image $A$ and image $B$, with $\mathrm{p}^{\mathrm{T}}{ }_{\mathrm{A}}$ and $\mathrm{p}^{\mathrm{T}} \mathrm{B}_{\mathrm{B}}$ representing marginal probability distributions.

$$
\begin{gathered}
H(A)=-\sum_{a} p_{A}^{T}(a) \log p_{A}^{T}(a) \quad \forall A\left(x_{A}\right)=a \mid x_{A} \in \Omega_{A, B}^{T} \\
H(B)=-\sum_{b} p_{B}^{T}(b) \log p_{B}^{T}(b) \quad \forall B^{T}\left(x_{A}\right)=b \mid x_{A} \in \Omega_{A, B}^{T}
\end{gathered}
$$

MMI is computed only by considering relationship between individual pixels in 2D image, with use of DTCWT sub bands MMI computed also considers spatial information. Optimization is the process of identifying the maximum or the minimum similarity measure between two images. Mathematically defined as min D $\left[\mathrm{MRC}^{1}{ }_{1 \mathrm{a} / \mathrm{b}}, \mathrm{T}\left(\mathrm{CTC}^{1}{ }_{1 \mathrm{a} / \mathrm{b}}\right)\right]$, where $\mathrm{T}$ is the transformation function. The optimizer metric based on gradient descent (Balci et al 2007, Tang et al 2006) is defined by Match

$($ MRI , CT $)=$ Sum (MRI CT $) / \sqrt{ }($ Su MRI $\cdot$ Su CT $)$, where Su MRICT $+=$ MRI( index $) \cdot \mathrm{CT}($ index $)$

and all other terms similarly defined. From the transformation parameters as represented in Eq. (5), the MRI data (every pixel at positions $\left\{\mathrm{x}_{\mathrm{r}}, \mathrm{y}_{\mathrm{r}}\right\}$ is transformed to obtain the registered image with respect to $\mathrm{CT}$ image represented by pixel positions $\left\{\mathrm{x}_{\mathrm{p}}, \mathrm{y}_{\mathrm{p}}\right\}$.

$$
\left[\begin{array}{c}
x_{p} \\
y_{p} \\
1
\end{array}\right]=\left[\begin{array}{ccc}
\mathrm{s} \cos \phi & -\mathrm{s} \sin \phi & \Delta \mathrm{x} \\
\mathrm{s} \sin \phi & \mathrm{s} \cos \phi & \Delta \mathrm{y} \\
0 & 0 & 1
\end{array}\right]\left[\begin{array}{c}
x_{r} \\
y_{r} \\
1
\end{array}\right]
$$

Where, $\phi$ represents the angle of rotation, s represents the scale factor, $\Delta \mathrm{x}$ and $\Delta \mathrm{y}$ represents the translation in $\mathrm{x}$ and $\mathrm{y}$ directions. With initial translation parameters set, MRI data is translated and compared with CT data by considering Mattes mutual information metric. Optimum transformation parameters are achieved with gradient descent algorithm with 300 iterations set as default parameter.

\section{Proposed work 3D image registration algorithm}

The proposed image registration algorithm is shown in Fi3. Registration is carried out by considering individual frames of the 3D data sets. The reconfigurable logic designed selects alternate frames of CT and MRI data and registration is carried out to obtain the corresponding frames. From the obtained even frames the odd frames are predicted based on bi-directional prediction algorithm. Prediction of odd number low pass bands is by considering the averaging method. In the averaging method, every pixel from the $\mathrm{n}^{\text {th }}$ frame and $(n+2)$ frame is averaged to obtain $n+1$ frame. For prediction of odd frames of high pass bands a novel algorithm is proposed.

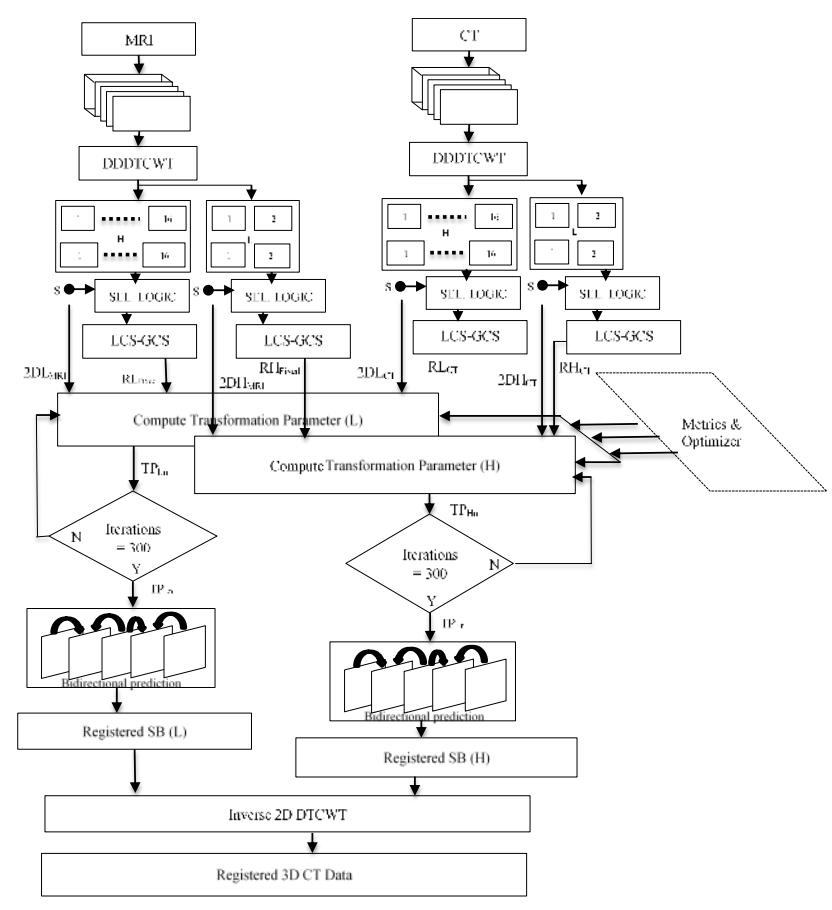

Fig. 3 Proposed 3D image registration algorithm

\subsection{High pass ba nd registration algorithm}

The high pass band registration algorithm considers the orientations feature and its significance of each pixel in the high pass sub band. From each of the real and imaginary registered even sub bands, the gradients (along the horizontal axis represented as in Eq. 6) in $\left\{ \pm 0^{0}, \pm 15^{0}, \pm 45^{0}\right.$, $\left.\pm 75^{0}\right\}$ are computed.

$$
\begin{aligned}
& \Delta_{0}^{0}=|\mathrm{x}(\mathrm{m}, 2 \mathrm{n}-1)-\mathrm{x}(\mathrm{m}, 2 \mathrm{n}+1)| \\
& \Delta_{180^{0}}=|\mathrm{x}(\mathrm{m}-1,2 \mathrm{n})-\mathrm{x}(\mathrm{m}+1,2 \mathrm{n})| \\
& \Delta_{45^{0}}=|\mathrm{x}(\mathrm{m}+1,2 \mathrm{n}-1)-\mathrm{x}(\mathrm{m}-1,2 \mathrm{n}+1)| \\
& \Delta_{135^{0}}=|\mathrm{x}(\mathrm{m}-1,2 \mathrm{n}-1)-\mathrm{x}(\mathrm{m}+1,2 \mathrm{n}+1)| \\
& \Delta_{15}{ }^{0}=|\mathrm{x}(\mathrm{m}+1,2 \mathrm{n}-1)-\mathrm{x}(\mathrm{m}, 2 \mathrm{n}+1)| \\
& \Delta_{165^{0}}=|\mathrm{x}(\mathrm{m}+1,2 \mathrm{n}-1)-\mathrm{x}(\mathrm{m}+1,2 \mathrm{n}+1)| \\
& \Delta_{75}{ }^{0}=|\mathrm{x}(\mathrm{m}+1,2 \mathrm{n}-1)-\mathrm{x}(\mathrm{m}-2,2 \mathrm{n}+1)| \\
& \Delta_{105^{0}}=|\mathrm{x}(\mathrm{m}-2,2 \mathrm{n}-1)-\mathrm{x}(\mathrm{m}+1,2 \mathrm{n}+1)|
\end{aligned}
$$




\begin{tabular}{l|l|l|l|l} 
& & & & \\
\hline$m-2,2 n-1$ & $m-2,2 n$ & $m-2,2 n+1$ & \\
\hline$m-1,2 n-1$ & $m-1,2 n$ & $m-1,2 n+1$ & \\
$-m, 2 n-1$ & $m, 2 n$ & $m, 2 n+1$ & \\
\hline$m+1,2 n-1$ & $m+1,2 n$ & $m+1,2 n+1$ & \\
\hline & & & &
\end{tabular}

Fig. 4 Registered Subband Image Segment

The prediction algorithm predicts the odd frame by considering pixel orientations in all 8 directions. Fig.4 represents the registered image segment to illustrate the novel prediction algorithm that is based on forward and backward prediction algorithm.

The forward prediction algorithm is presented in Fig. 5, and represented by Eq. (7) the pixel position $\tilde{y}(m, 2 n)$ is computed by considering the pixels in Frame $F_{n}$ and Frame $\mathrm{F}_{\mathrm{n}+2}$. As there are 8 orientations, eight intermediate frames are predicted by considering the $F_{n}(m, 2 n)$ as the reference pixel position.

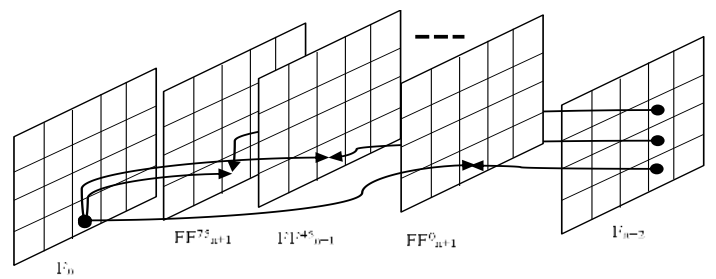

Fig.5 Forward prediction algorithm

The forward prediction algorithm computes every pixel in the intermediate frame, with each intermediate frame comprising of 8 orientations along the direction $\left\{ \pm 0^{0}(0\right.$ \& $180), \pm 15^{\circ}(15 \& 165), \pm 45^{0}$ (45\&135), $\left.\pm 75^{0}(75 \& 105)\right\}$.

$\tilde{y}_{0}^{0}(\mathrm{~m}, 2 \mathrm{n})=[\mathrm{x}(\mathrm{m}, 2 \mathrm{n}-1)+\mathrm{y}(\mathrm{m}, 2 \mathrm{n}+1)] / 2$,

$\tilde{y}_{180} 0(\mathrm{~m}, 2 \mathrm{n})=[\mathrm{x}(\mathrm{m}-1,2 \mathrm{n})-\mathrm{y}(\mathrm{m}+1,2 \mathrm{n})] / 2$

(7a)

$\tilde{y}_{45}{ }^{0}(\mathrm{~m}, 2 \mathrm{n})=[\mathrm{x}(\mathrm{m}+1,2 \mathrm{n}-1)+\mathrm{y}(\mathrm{m}-1,2 \mathrm{n}+1)] / 2$

$\tilde{y}_{135^{\circ}}(\mathrm{m}, 2 \mathrm{n})=[\mathrm{x}(\mathrm{m}-1,2 \mathrm{n}-1)+\mathrm{y}(\mathrm{m}+1,2 \mathrm{n}+1)] / 2$

$\tilde{y}_{15}{ }^{0}(\mathrm{~m}, 2 \mathrm{n})=[\mathrm{x}(\mathrm{m}+1,2 \mathrm{n}-1)+\mathrm{y}(\mathrm{m}, 2 \mathrm{n}+1)] / 2$

$\left.\tilde{y}_{165^{0}}(\mathrm{~m}, 2 \mathrm{n})=[\mathrm{x}(\mathrm{m}+1,2 \mathrm{n}-1)+\mathrm{y}(\mathrm{m}+1,2 \mathrm{n}+1)]\right] / 2$

$\tilde{y}_{75}{ }^{0}(\mathrm{~m}, 2 \mathrm{n})=[\mathrm{x}(\mathrm{m}+1,2 \mathrm{n}-1)+\mathrm{y}(\mathrm{m}-2,2 \mathrm{n}+1)] / 2$

$\tilde{y}_{105}{ }^{0}(\mathrm{~m}, 2 \mathrm{n})=[\mathrm{x}(\mathrm{m}-2,2 \mathrm{n}-1)+\mathrm{y}(\mathrm{m}+1,2 \mathrm{n}+1)] / 2$

Backward prediction algorithm is presented in Fig.6 and the computation of each pixel in the intermediate frame is computed as in Eq. (8), by considering the pixel in Frame $\mathrm{F}_{\mathrm{n}+2}$ as the reference frame.

$\hat{\mathrm{z}}_{0}{ }^{0}(\mathrm{~m}, 2 \mathrm{n})=[\mathrm{y}(\mathrm{m}, 2 \mathrm{n}-1)+\mathrm{x}(\mathrm{m}, 2 \mathrm{n}+1)] / 2$,

$\hat{\mathrm{z}}_{180} 0^{\circ}(\mathrm{m}, 2 \mathrm{n})=[\mathrm{y}(\mathrm{m}-1,2 \mathrm{n})-\mathrm{x}(\mathrm{m}+1,2 \mathrm{n})] / 2$

$\hat{\mathrm{Z}}_{45^{\circ}}(\mathrm{m}, 2 \mathrm{n})=[\mathrm{y}(\mathrm{m}+1,2 \mathrm{n}-1)+\mathrm{x}(\mathrm{m}-1,2 \mathrm{n}+1)] / 2$

$\hat{\mathrm{z}}_{135^{\circ}}(\mathrm{m}, 2 \mathrm{n})=[\mathrm{y}(\mathrm{m}-1,2 \mathrm{n}-1)+\mathrm{x}(\mathrm{m}+1,2 \mathrm{n}+1)] / 2$

$\hat{\mathrm{z}}_{15}{ }^{0}(\mathrm{~m}, 2 \mathrm{n})=[\mathrm{y}(\mathrm{m}+1,2 \mathrm{n}-1)+\mathrm{x}(\mathrm{m}, 2 \mathrm{n}+1)] / 2$ $\left.\hat{\mathrm{z}}_{165^{0}}(\mathrm{~m}, 2 \mathrm{n})=[\mathrm{y}(\mathrm{m}+1,2 \mathrm{n}-1)+\mathrm{x}(\mathrm{m}+1,2 \mathrm{n}+1)]\right] / 2$

$\hat{\mathrm{z}}_{75}{ }^{0}(\mathrm{~m}, 2 \mathrm{n})=[\mathrm{y}(\mathrm{m}+1,2 \mathrm{n}-1)+\mathrm{x}(\mathrm{m}-2,2 \mathrm{n}+1)] / 2$

$\hat{\mathrm{z}}_{105^{0}}(\mathrm{~m}, 2 \mathrm{n})=[\mathrm{y}(\mathrm{m}-2,2 \mathrm{n}-1)+\mathrm{x}(\mathrm{m}+1,2 \mathrm{n}+1)] / 2$

The forward and backward prediction algorithm results in frames $\mathrm{FF}_{\mathrm{n}}{ }_{\mathrm{n}}(\mathrm{m}, 2 \mathrm{n})$ and $\mathrm{FB}_{\mathrm{n}}{ }_{\mathrm{n}}(\mathrm{m}, 2 \mathrm{n})$ frames respectively, the average of these frames $\left(\mathrm{FP}_{n}{ }_{n}(\mathrm{~m}, 2 \mathrm{n})=\left[\mathrm{FB}^{\Theta}{ }_{\mathrm{n}}(\mathrm{m}, 2 \mathrm{n})+\right.\right.$ $\left.\left.\mathrm{FF}_{\mathrm{n}}{ }_{\mathrm{n}}(\mathrm{m}, 2 \mathrm{n})\right] / 2\right)$ are computed to obtain the intermediate frames in all eight orientations. As DD DTCWT results in localization of orientations and features in each of the sub bands, the best feature and orientation is computed for the intermediate frame by considering all the features obtained by the prediction algorithm as in Eq. (9),

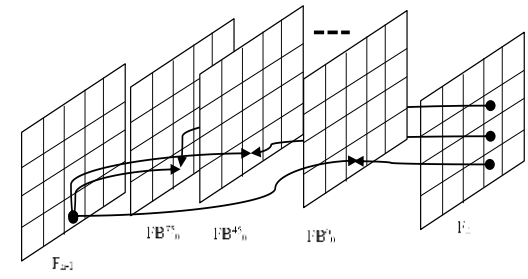

Fig. 6 Backward prediction algorithm

$\mathrm{FP}_{n}(\mathrm{~m}, 2 \mathrm{n})=$ Mean $\left\{\mathrm{FB}^{0}{ }_{\mathrm{n}}(\mathrm{m}, 2 \mathrm{n}), \mathrm{FB}^{15}{ }_{\mathrm{n}}(\mathrm{m}, 2 \mathrm{n}), \mathrm{FB}^{45}{ }_{\mathrm{n}}\right.$ $(\mathrm{m}, 2 \mathrm{n}), \mathrm{FB}^{75} \mathrm{n}(\mathrm{m}, 2 \mathrm{n})$ and $\left.\mathrm{FB}^{180}{ }_{\mathrm{n}}(\mathrm{m}, 2 \mathrm{n})\right\}$

The predicted low pass band and the predicted high pass bands are combined and inverse transform is computed. The registered $3 \mathrm{D}$ image by considering $2 \mathrm{D}$ data sets is registered by considering the data features computed by considering all the sub bands of DD DTCWT with minimum redundancy.

\section{Results \& Discussion}

The proposed algorithm is modeled in MATLAB and is evaluated for its performance by considering standard data sets.
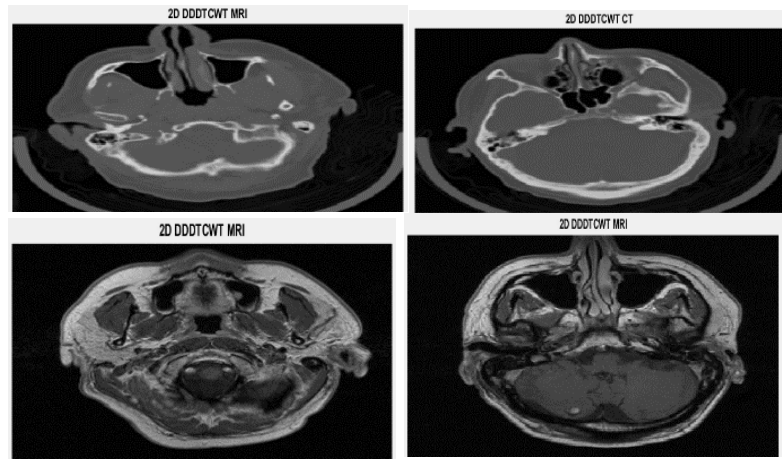


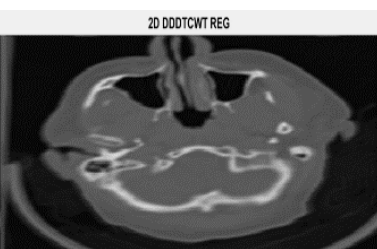

(a)

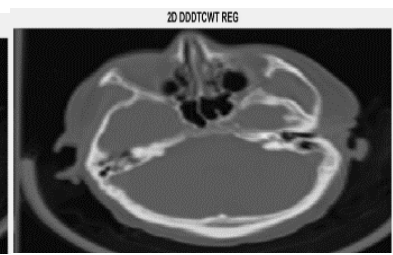

(b)

Fig. 7 Registered image (a) Frame 1 (b) Frame 16

Fig. 7 presents the results of two frames $\left(1^{\text {st }}\right.$ and $16^{\text {th }}$ frame $)$ of CT, MRI and Registered image. The registration process is efficient as it is able to capture information in CT and MRI data effectively and transformation is carried out to obtain the registered image. Fig. 8 presents the metrics of individual frames indicating mutual information and joint entropy metric of registered 3D data of the frames 1, 2, 4, 8,24 and 32. From the metrics obtained it is found that the proposed registration process is able to register the input data and is indicated by the MI and JE metric with maximum variation of $56 \%$ and $4.8 \%$ respectively.
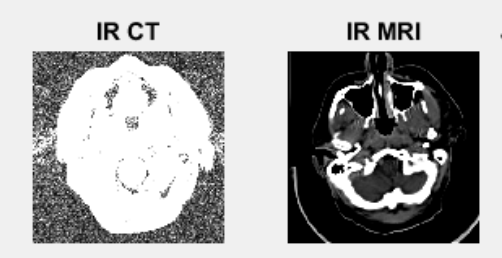

(a) $\mathrm{MI1}=\mathbf{- 0 . 5 0 2 2}$ and $\mathrm{JE} 1=7.4577$
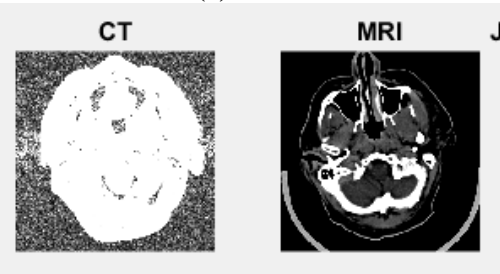

Joint Histogram before IR

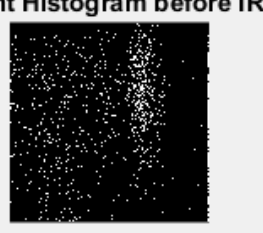

(b) $\mathrm{MI2}=\mathbf{- 0 . 5 0 5 2}$ and JE2 $=\mathbf{7 . 4 7 5 7}$

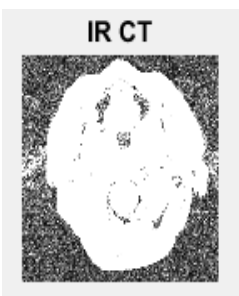
IR MRI

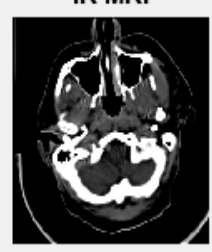

(c) $\mathrm{MI} 4=-\mathbf{0 . 6 0 9 4}$ and $\mathrm{JE} 4=\mathbf{7 . 9 0 7 8}$
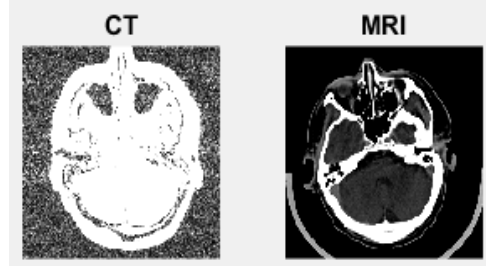

Joint Histogram before IR

(d) $\mathrm{MI8}=-\mathbf{0 . 7 3 1 4}, \mathrm{JES}=7.8575$

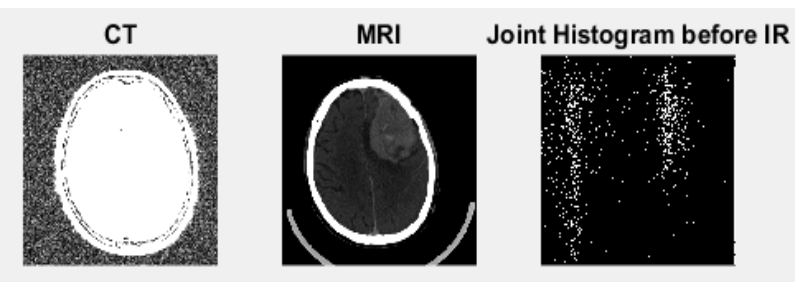

(e) MI24 $=\quad 0.2651$ and JE24 $=8.0455$

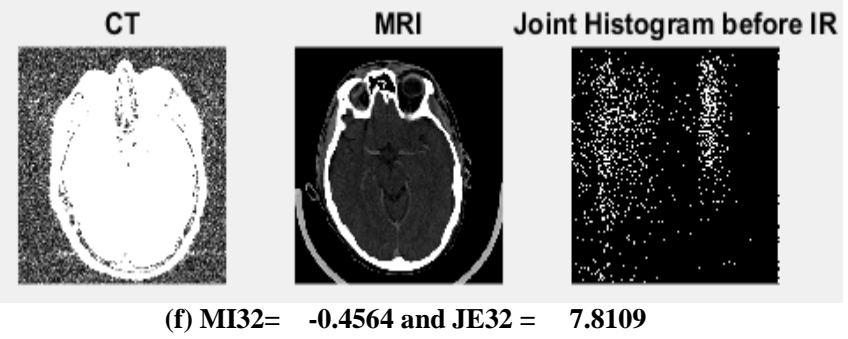

Fig. 8 (a-f) Image Registration Metrics

Table 2 compares the MI and JE metrics of registered and unregistered images of all 10 patients. From the comparisons it is found that the cumulative MI is 8.2525 higher than $\mathrm{MI}$ in unregistered data and the $\mathrm{JE}$ is $6 \%$ higher than unregistered image.

Table 2 Comparison of IR Metrics

\begin{tabular}{|c|c|c|c|c|}
\hline Patient & $\begin{array}{c}\text { JE before } \\
\text { IR }\end{array}$ & JE after IR & $\begin{array}{c}\text { MI } \\
\text { before } \\
\text { IR }\end{array}$ & $\begin{array}{c}\text { MI after } \\
\text { IR }\end{array}$ \\
\hline ID 001 & 250.8237 & 252.52221 & -5.9333 & 2.3192 \\
\hline ID 002 & 178.1112 & 189.09810 & -2.9022 & 3.0091 \\
\hline ID 003 & 064.4490 & 071.11191 & -8.0012 & 1.2891 \\
\hline ID 004 & 351.9834 & 356.80987 & 1.2103 & 8.0914 \\
\hline ID 005 & 289.9871 & 294.09101 & 2.5079 & 11.9010 \\
\hline ID 006 & 155.1478 & 157.12890 & -5.7061 & 3.9012 \\
\hline ID 007 & 198.7251 & 201.72891 & -2.0001 & 5.0901 \\
\hline ID 008 & 267.9031 & 269.09823 & -9.9097 & 1.2783 \\
\hline ID 009 & 180.1781 & 184.90871 & -5.1110 & 2.4723 \\
\hline ID 010 & 368.9087 & 371.00182 & -4.9092 & 3.5234 \\
\hline
\end{tabular}

\section{Conclusions}

Image registration using DD DTCWT is demonstrated to have achieved registration metric of mutual information by 10 times and joint entropy by more than $6 \%$ by considering the redundant and non-redundant information from wavelet sub bands. The proposed algorithm estimates the features from the sub bands and the transformation process is carried out to maximize mutual information in the two images to be registered. Use of reconfigurable logic with selection of sub bands ensures use of proposed registration algorithm for real time applications. 


\section{References}

[1] Y. P. Shen, "Review of image registration methods for medical images," Chinese Journal of Medical Physics, vol. 30, no. 1, pp. 3885-3889, 2013.

[2] M. Toews and W.M. Wells III, "Efficient and robust model-to-image alignment using 3D scale-invariant features," Medical Image Analysis, vol. 17, no. 3, pp. 271-282, 2013.

[3] F. P.M.Oliveira and J. M. R. S. Tavares, "Medical image registration: a review," ComputerMethods in Biomechanics and Biomedical Engineering, vol. 17, no. 2, pp. 73-93, 2014

[4] Maha Abdul - Rahman Hasso and Ruaa Moayad Elya "Fast Image Registration Based on Features Extraction and Accurate Matching Points for Image Stitching" IJCSI International Journal of Computer Science Issues, Vol. 11, Issue 5, No 1, September 2014 pp138-142

[5] Jharna Majumdar, Vanathy.B and Lekshmi. "Iterative Refinement of Transformation Parameters for Image registration " IJCSI International Journal of Computer Science Issues, Vol. 7, Issue 5, September 2010 pp262-267

[6] J.Zheng,Z. Ji, K. Yu,Q.An, Z. Guo, andZ.Wu, "A feature-based solution for $3 \mathrm{D}$ registration of $\mathrm{CT}$ and MRI images of human knee," Signal, Image and Video Processing, vol. 9, no. 8, pp. 1815-1824, 2015.

[7] Zhiying Song, Huiyan Jiang, Qiyao Yang, Zhiguo Wang,2 and Guoxu Zhang, "A Registration Method Based on Contour Point Cloud for 3D Whole-Body PET and CT Images", Hindawi BioMed Research International, Volume 2017, Article ID 5380742, 11 pages.

[8] Milad Ghantous, Somik Ghosh, Magdy Bayoumi, A MULTI-MODAL AUTOMATIC IMAGE REGISTRATION TECHNIQUE BASED ON COMPLEX WAVELETS

[9] Jingjie Zheng · Zhenyan Ji · Kuangdi Yu · Qin An -Zhiming Guo · Zuyi Wu, A feature-based solution for 3D registration of CT and MRI images of human knee, DOI 10.1007/s11760-014-0660-5

[10] A. Grossman and J. Morlet, Decomposition of hardy functions into square integrable wavelets of constant shape, SIAM J. Math. Anal., 15(4), 723-736, 1984

[11] S. Mallat, Multiresolution approximation and wavelets, Trans. Of American Math. Soc., 315, 69-88, 1989

[12] I. Daubechies, Orthonormal bases of compactly supported wavelets II, variation on a theme, SIAM Journal of Mathematical Analysis, 24(2), 499-519, March 1993

[13] G. Strang, Wavelets and dilation equations: A brief introduction, SIAM Review, 31 (4), 614-627, 1989

[14] A. Grossman, R. Kronland-Martinet and J. Morlet, "Reading and understanding continuous wavelet transforms", In J. M. Combes, A. Grossman, and Ph.
Tchmaitchian, editors, Wavelets Time Frequency Methods and Phase Space, pages 2-20, Berlin, 1989, Springer-Verlag. Proceedings of International Colloquium on wavelets and Applications, Marseille, France, December 1987

[15] G. H. Granlund and H. Knutsson, Signal Processing for computer Vision, Kluwer Academic Publisher, 1995]

[16] S. Mallat, Zero crossing of a wavelet transform, IEEE Trans. Information Theory, 37(4), July 1991

[17] N. G. Kingsbury and J. F. A. Magarey, Wavelet transform in image processing, in Proc. First European Conference on Signal Analysis and Prediction, 23-34, Prague, June 24-27, 1997

[18] Felix C. A. Fernandes, Directional, Shift-Insensitive, Complex Wavelet Transforms with Controllable Redundancy, PhD Thesis, RICE university, Houston, Texas, 2002

Girija J, awarded Bachelors of Engineering from. Bangalore University in 1995, M. Tech. from VTU in 2000, pursing Ph.D. in JNTU Anantapuram Andrapradesh , currently Working as Associate Professor in Department of Computer Science \& Engg. Bangalore Institute of Technology, Bangalore. Having teaching experience of about 17 year in BIT, Bangalore. Worked as a project assistant at Indian Institute of Science under Prof. Soundranayagam. Presented paper in many national and international conferences and published journals in IJEECS, and IEEE explorer etc.,

Dr. G. N. KrishnaMurthy. Ph.D, M.Tech.,B.E., presently working as Principal BNMIT Bangalore Published many conference and journals.

Dr. P. Chenna Reddy. Ph.D, M.Tech., B.E, presently working as Director of Academic Audit JNTUA Ananthapuram, AP. Published many conference and journals. 